\title{
WEBQUEST CREATOR: Ferramenta para Criação de WebQuests
}

Jader Bittencourt, FACIN/PUCRS - jaderbittencourt@gmail.com

Márcia Cristina Moraes, FACIN/PUCRS - mmoraes@pucrs.br

Resumo. Este artigo tem como objetivo apresentar uma ferramenta para a criação de WebQuests no Moodle. Esta ferramenta se diferencia das atuais na medida em que possui novas funcionalidades como: a customização de layouts para a página da WebQuest; ampliação da estrutura básica de introdução, tarefas, processos, recursos, avaliação e conclusão para inclusão de novos elementos; e atualização automática da estrutura básica quando da inserção de novos elementos. Esperamos com esta ferramenta facilitar e agilizar a criação e customização de WebQuests.

Palavras-chaves: Webquests, customização de layouts, Moodle

\section{WEBQUEST CREATOR: Tool for WebQuest Creation}

Abstract. This article aims to present a tool for creating WebQuests in Moodle. This tool differs from the current ones because it has new features such as customizing page layouts for the WebQuest; expansion of the basic structure of introduction, tasks, processes, resources, evaluation, and conclusion to include new elements, and automatic update the basic structure when the insertion of new elements. We expect that this tool will facilitate the creation and customization of WebQuests.

Keywords: Webquests, layout customization, Moodle

\section{Introdução}

Hoje em dia nossa sociedade vive uma constante evolução tecnológica. Esta evolução acabou por se tornar tão corriqueira que muitas vezes passa quase que despercebida aos nossos olhos. Nossos celulares, que antes serviam apenas para telefonar e mandar mensagens de texto, hoje tocam música, possuem rádio, tiram fotos, etc. Compramos televisores de LCD, com uma alta definição de imagem e som, efetuamos compras através da internet e muitas vezes os produtos se encontram do outro lado do mundo, pagamos nossas contas e fazemos transações bancárias sem mesmo sair de casa. Tudo isso é a tecnologia que nos cerca, e que já faz parte de nossas vidas.

Juntamente com esta evolução tecnológica temos a inclusão digital, que vem se tornando, a cada dia, uma realidade maior em nossa sociedade. Hoje, como conseqüência da constante redução no valor dos computadores e do acesso à Internet, se torna cada vez mais fácil que as classes sociais, que outrora não tinham condição de adquirir estes tipos de produtos, acabem por adquiri-los. ¿̀ medida que esta democratização do acesso à tecnologia da informação vem aumentando, podemos assumir também que a imersão dos usuários da Internet cresce proporcionalmente.

Esse crescimento, aliado a necessidade de qualificação profissional, tem impulsionado os cursos em Educação a Distância (EAD). Estes cursos, em especial, utilizam práticas pedagógicas que envolvem o aluno na construção do conhecimento através de recursos disponíveis na Internet. Uma prática que pode ser utilizada para proporcionar um aprendizado significativo para os alunos é a WebQuest. A Webquest é 
uma investigação orientada, que possui um formato de aula, em que a maioria ou todas as informações com que os alunos trabalham é proveniente da Internet (Mercado e Viana, 2004).

Neste artigo iremos apresentar uma ferramenta para criação de Webquests chamada Webquest Creator (WQC). Esta ferramenta se diferencia das atuais na medida em que possui novas funcionalidades como: a customização de layouts para a página da WebQuest; ampliação da estrutura básica de introdução, tarefas, processos, recursos, avaliação e conclusão para inclusão de novos elementos; e atualização automática da estrutura básica quando da inserção de novos elementos. A ferramenta foi desenvolvida como um plugin para o Moodle. O Moodle é Ambiente Virtual de Aprendizagem projetado para dar suporte a teoria do construcionismo social (Moodle, 2011).

O artigo está organizado em cinco seções. A seção dois apresenta o referencial teórico sobre WebQuest. A seção 3 descreve os trabalhos relacionados. A seção 4 apresenta a ferramenta Webquest Creator (WQC). A seção 5 apresenta as considerações finais.

\section{WebQuest}

A tecnologia educacional WebQuest foi proposta em 1995, por Bernie Dodge, professor da San Diego State University, e destina-se à educação presencial, com participação ativa dos alunos sob a orientação do professor, estendendo-se pela pesquisa guiada na Internet. Uma atividade WebQuest oferece a possibilidade da construção do saber em um processo cooperativo na realização de um projeto (Abar e Barbosa 2008).

Por padrão uma página Webquest possui uma estrutura pré-definida, que consiste basicamente em (Mercado e Viana, 2004):

- Introdução: descreve uma breve introdução em relação à proposta oferecida pela Webquest e também a origem do projeto em alguns casos;

- Tarefas: apresenta os objetivos e atividades a serem realizadas na Webquest;

- Processos: descreve uma seqüência de passos a serem seguidos para que a WebQuest seja realizada;

- Recursos: indica os recursos disponíveis para consulta e solução da Webquest;

- Avaliação: descreve como será realizada a avaliação do projeto proposto pela WebQuest;

- Conclusão: apresenta uma conclusão do ponto de vista de seus autores em relação a uma possível solução da WebQuest. Relata como foi o desenrolar das atividades exercidas durante $\mathrm{o}$ período em que os envolvidos estiveram dedicados para conclúrem cada um dos passos da Webquest.

Variações deste estilo padrão podem ser vistas em algumas Webquests que possuem uma estrutura um pouco maior, com informações como Orientação e Autores.

\section{Trabalhos Relacionados}

Atualmente muitos módulos e plugins já foram desenvolvidos e compartilhados para o Moodle, e estes podem ser facilmente encontrados na Internet e instalados com o auxílio de tutoriais. Um destes é o Moodle Module Webquest (2010) que tem como objetivo a criação de atividades WebQuest, porém, segundo pesquisa realizada no fórum do Moodle referente a este módulo, o mesmo não se encontra totalmente funcional. Outro trabalho relacionado propõe a criação de um curso no Moodle 
estruturado como uma Webquest (Miguel, 2010). A proposta é interessante, mas difere em termos de funcionalidade da proposta oferecida pelo WebQuest Creator (WQC).

Diferentemente do plugin Moodle Module Webquest, o WQC oferece a possibilidade de uma customização de layouts e da sua estrutura da WebQuest, possibilitando inserir novos elementos à estrutura básica. Para melhor visualização, na tabela 1 estão ilustradas as diferenças entre funcionalidades oferecidas pelo Moodle Module Webquest e pelo WQC. Cabe salientar que o trabalho de Miguel (2010) não foi inclú́do na tabela pelo fato de não se tratar especificamente de um módulo, mas sim, um curso estruturado na forma de Webquest.

Tabela 1 - Diferença de funcionalidade entre o Moodle Module WebQuest e o WQC

\begin{tabular}{|l|c|c|}
\hline \multicolumn{3}{|c|}{ Diferenças entre os módulos } \\
\hline \multicolumn{1}{|c|}{ Funcionalidades } & Moodle Module Webquest & WQC \\
\hline Cadastro de Webquests & & $\checkmark$ \\
\hline Cadastro e edição de conteúdos na estrutura báscia & & $\checkmark$ \\
\hline Status: visivel / oculto & & $\checkmark$ \\
\hline Customização dos conteúdos da Webquest & $\mathbf{X}$ & \\
\hline Inclusão de novos elementos na estrutura da Webquest & $\mathbf{X}$ & \\
\hline Inclusão de conteúdo em novos elementos & $\mathbf{K}$ & \\
\hline Cadastro e edição de Layouts & $\mathbf{K}$ & \\
\hline Customização de Layouts & $\mathbf{X}$ & \\
\hline Atualização automática da estrutura básica com novos elementos & & \\
\hline
\end{tabular}

Como pode ser visto, o WQC oferece funcionalidades relacionadas à customização de layouts e inclusão de elementos na estrutura básica da Webquest, o que não é oferecido pelo Moodle Module Webquest.

\section{WebQuest Creator}

O WebQuest Creator (WQC) é uma ferramenta, desenvolvida como um plugin para o Moodle, que possibilita a criação de Webquests. O objetivo da ferramenta é estabelecer uma padronização mínima para a criação de WebQuests, facilitando o entendimento e interpretação do processo de produção de uma WebQuest.

A ferramenta é composta por três módulos: layouts (responsável pelo cadastro e gerenciamento dos layouts que poderão ser vinculados as Webquests); elementos (consiste no cadastro e edição de elementos para a estrutura da Webquest); e WebQuests (responsável pela montagem da WebQuest através da seleção do layout e elementos a serem utilizados na atividade).

Nas próximas seções serão apresentadas descrições detalhadas de cada um destes módulos e as questões relacionadas a implementação da ferramenta.

\subsection{Módulo Layouts}

Este módulo é responsável pelo gerenciamento dos layouts que são vinculados as WebQuests. O cadastro dos layouts acontece em duas etapas, sendo a primeira um cadastro básico, onde o usuário insere como informações básicas o nome do layout, um status, escolhendo entre ativo e inativo e duas cores, sendo uma cor para a fonte que será vinculada aos textos e outra cor para o background. O usuário também tem a 
possibilidade de inserir uma imagem para ser exibida no topo da WebQuest, sendo esta opcional. No caso do usuário não inserir uma imagem como headder da WebQuest, o sistema automaticamente exibe o nome da Webquest no topo da página, logo abaixo da barra do Moodle. A figura 1 apresenta o formulário utilizado no credenciamento dos layouts.

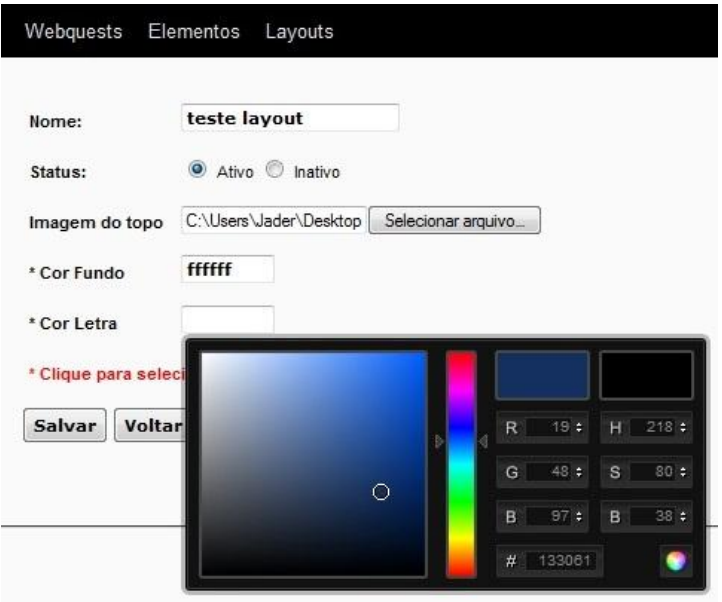

Figura 1. Formulário de Layout

Em um segundo momento, o usuário tem a possibilidade de cadastrar botões (intitulados botão A e botão B) para os elementos e vinculá-los a um determinado layout previamente cadastrado. O botão A é exibido por default, ou seja, ele é exibido sempre que o respectivo elemento do botão não está selecionado na visualização da WebQuest. O botão B por sua vez tem a intenção de efeito visual, isto é, quando o usuário que estiver visualizando a WebQuest passar o mouse sobre o elemento, a ferramenta efetua a troca da imagem exibida automaticamente da imagem A para a imagem B, realizando assim o efeito. Quando um elemento da Webquest está selecionado para visualização, o botão que é exibido por padrão é o botão $\mathrm{B}$, fazendo assim com que o elemento selecionado tenha destaque em relação aos demais. $\mathrm{O}$ formulário de cadastro dos elementos de layout pode ser visualizado na figura 2 .

\section{Webquests Elementos Layouts}

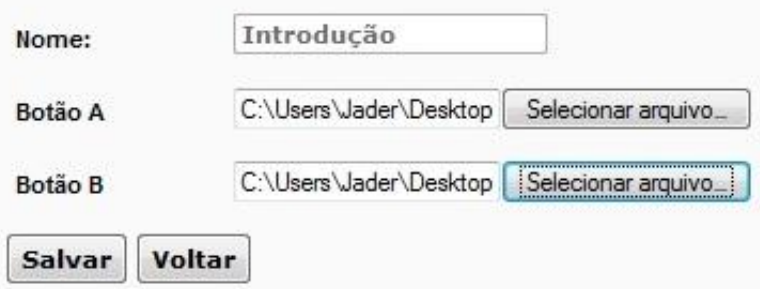

Figura 2. Formulário de Elementos de Layout

Apenas layouts ativos podem ser vinculados a WebQuests. É obrigatório que sejam inseridos ao menos todos os elementos básicos de uma WebQuest para o layout, ou seja, caso o usuário cadastre o elemento Introdução a um layout $X$, ele será automaticamente obrigado a inserir todos os outros elementos básicos para este mesmo 
layout e também é obrigado a inserir os botões A e B para eles. Esta obrigatoriedade se dá pelo fato de que uma Webquest possui no mínimo os elementos básicos (Introdução, Tarefas, Processos, Recursos, Avaliação e Conclusão) vinculados a ela. Se um layout estiver sendo utilizado por uma Webquest e este layout não possuir todos os elementos básicos vinculados a ele, caso exista pelo menos um único elemento básico cadastrado no layout, isso fará com que o layout fique inconsistente na hora da exibição da WebQuest, pois neste momento seriam exibidos os botões A e B apenas para este elemento, fazendo com que os demais elementos fossem exibidos como links (figura 3).

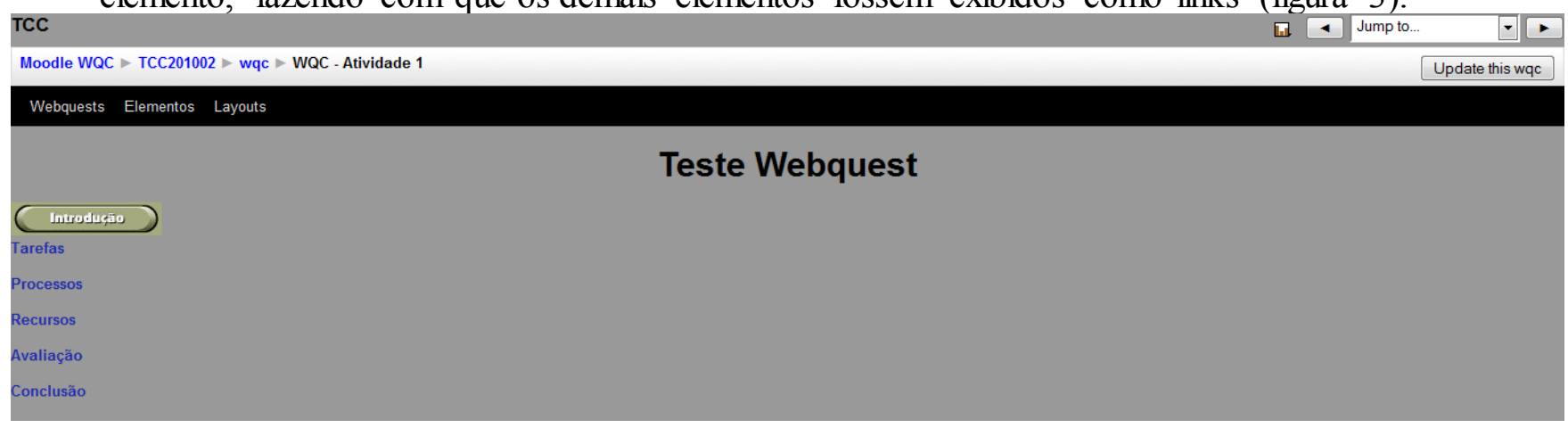

Figura 1 Layout com um único elemento cadastrado

\subsection{Módulo Elementos}

Este módulo é responsável pelos elementos que podem ser vinculados as WebQuests. Por padrão, a ferramenta já traz cadastrados os elementos: Introdução, Tarefas, Processos, Recursos, Avaliação e Conclusão. Estes, por se tratarem dos elementos básicos, não ficam disponíveis para edição, pois não podem ser alterados e nem excluídos.

Inicialmente o usuário visualiza uma tabela vazia informando que não existem elementos cadastrados. No momento de inserir um novo elemento o usuário precisa informar um nome para este, que também será o nome exibido no momento da visualização da WebQuest e um status, podendo escolher entre ativo e inativo. Elementos inativos não podem ser visualizados em nenhum local da ferramenta, exceto na própria área destinada a eles, que é a área de cadastro de elementos. Um elemento inativo não pode ser vinculado a nenhuma Webquest e/ou Layout. A figura 4 ilustra $o$ formulário de cadastro de elementos.

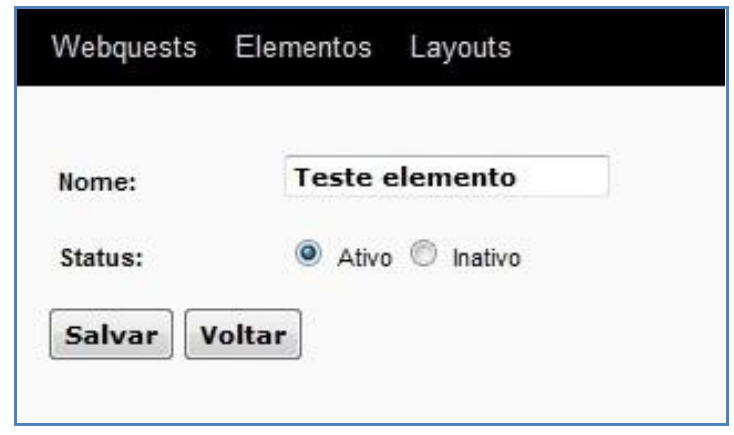

Figura 4 - Formulário de elementos

Os elementos ativos por sua vez podem ser visualizados em qualquer lugar da ferramenta. Cada elemento pode ser associado apenas uma única vez a uma WebQuest. Caso este seja excluído da WebQuest ele poderá ser inserido novamente caso seja da 
vontade do usuário. Se uma WebQuest possui um elemento e este tem seu status atualizado para inativo, ele não é removido da WebQuest, porém, não é possível visualizá-lo para edição, visualização, etc. Os elementos podem ser cadastrados para várias WebQuests.

\subsection{Módulo WebQuests}

Este é o módulo principal da ferramenta, concentrando o maior número disponível de ações. Este módulo possui, além da possibilidade de inserir novas WebQuests, as seguintes ações disponíveis: Editar, Elementos, Ordenar, Publicar e Visualizar.

O formulário de cadastro de WebQuests é relativamente semelhante ao formulário de cadastro de Elementos (figura 5).

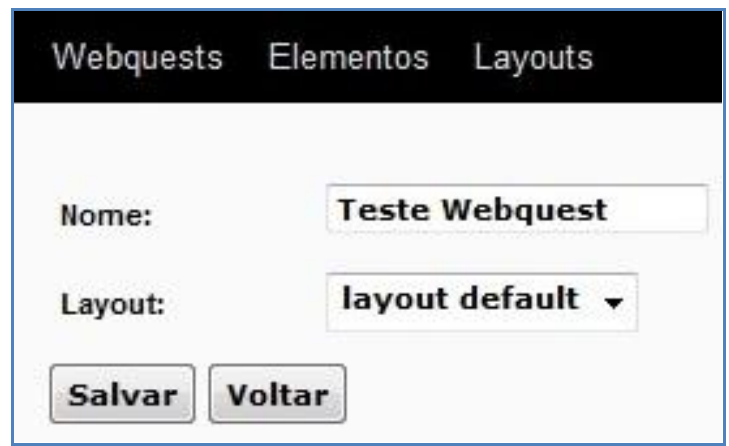

Figura 5. Formulário de webquests

A seguir temos as demais ações deste módulo e uma breve descrição de cada uma. A ação de "Editar" complementa a inserção de novas WebQuests e possui basicamente a possibilidade de inserir um nome para a WebQuests, que será exibido no topo da página, logo abaixo da barra do Moodle caso o layout selecionado não possua um headder, e uma lista com os layouts disponíveis para o cadastro, sendo estes apenas layouts como o status de ativo.

No momento em que uma nova WebQuest é inserida, o WQC automaticamente insere todos os elementos básicos nela, fazendo assim com que todas as WebQuests sigam o padrão básico de terem vinculadas a elas estes elementos. Os elementos básicos não podem ser excluídos de nenhuma Webquest. $\mathrm{Na}$ ação "Elementos" o usuário tem a possibilidade de inserir novos elementos na Webquest, de exclú-los e também de editar os seus conteúdos (figura 6). 


\section{Webquests Elementos Layouts}

Inserir novo Elemento na Webquest: Teste Webquest

\begin{tabular}{|c|c|c|}
\hline & \multicolumn{2}{|c|}{ Açōes } \\
\hline & Elemento & Editar | Excluir \\
\hline Introdução & Editar | Excluir \\
\hline Tarefas & Editar | Excluir \\
\hline Processos & Editar | Excluir \\
\hline Recursos & Editar | Excluir \\
\hline Avaliação & Editar | Excluir \\
\hline Conclusão & & \\
\hline
\end{tabular}

Figura 6. Lista de elementos da Webquest

No momento de inserir um novo elemento, é apresentada ao usuário uma lista com todos os elementos ativos disponíveis e que ainda não foram vinculados a Webquest.

Para a edição, a ferramenta carrega o TinyMCE, um aplicativo do PHP que fornece um editor de textos com várias opções de edição e customização, facilitando assim a criação dos conteúdos das Webquests.

Após inserir todo o conteúdo desejado através do editor de textos e clicar em salvar, o WQC exibe uma mensagem de confirmação. Ao confirmar a mensagem clicando em "ok" ele direciona novamente para a lista de elementos.

$\mathrm{Na}$ ação de "Ordenar" a ferramenta possibilita a customização da ordem em que os elementos vinculados a WebQuest serão exibidos no momento em que esta estiver sendo visualizada. A ferramenta ordena os elementos em sua ordem padrão, que é a mesma ordem já citada para os elementos básicos. Com esta funcionalidade, o usuário simplesmente informa um número que será utilizado pela ferramenta para ordenar os elementos na hora em que estes forem exibidos. Os números devem ser informados de forma crescente, sendo os números menores para os elementos que devem ser exibidos primeiro e os números maiores para os que devem ser exibidos por último, conforme figura 7 .

\section{Webquests Elementos Layouts}

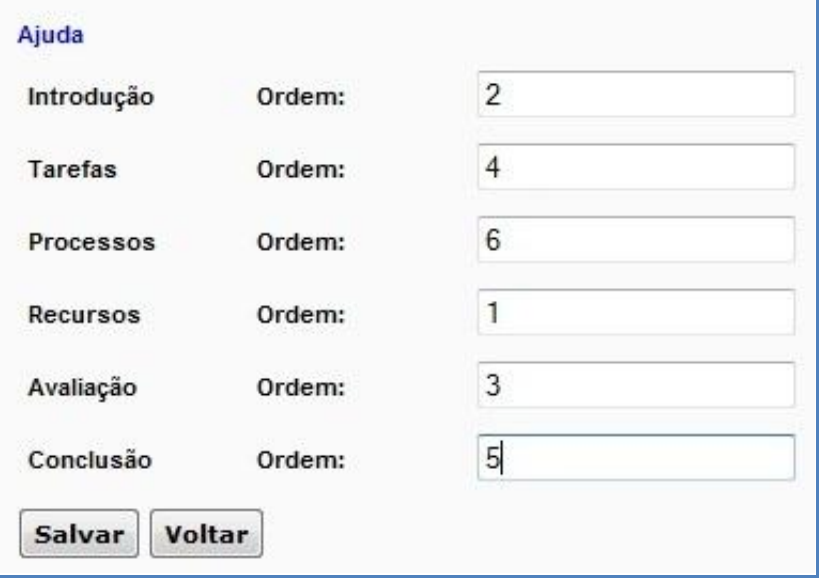

Figura 7. Formulário de ordenação de elementos 
Caso o usuário tenha dificuldade para compreender o funcionamento da ação ordenar, o WQC fornece uma ajuda que oferece ao usuário um exemplo prático de como esta ação deve ser utilizada. Após a ordenação, o WQC exibirá os elementos na ordem escolhida pelo usuário, tanto para edição dos elementos como para a visualização e publicação da WebQuest. Os elementos re-ordenados estão ilustrados na figura 8 .

\begin{tabular}{|l|l|l|}
\hline \multicolumn{2}{|l|}{ Açöes } \\
\hline & Elemento & Editar | Excluir \\
\hline & Recursos & Editar | Excluir \\
\hline Introdução & Editar | Excluir \\
\hline Avaliação & Editar | Excluir \\
\hline Tarefas & Editar | Excluir \\
\hline Conclusão & Editar | Excluir \\
\hline Processos & & \\
\hline
\end{tabular}

Figura 8. Elementos de webquest re-ordenados

A ação "Publicar" é responsável por publicar a Webquest. O ato de publicar uma Webquest consiste em alterar o seu status de "Não Publicada" para "Publicada" e vincular uma data a sua publicação. Para fazer isto, a ferramenta faz uma série de validações para que a WebQuest possa ser considerada apta para ser publicada. Para que uma Webquest possa ser publicada ela deve atender as seguintes exigências:

1 - Todos os elementos ativos vinculados a ela devem possuir um conteúdo cadastrado;

2 - O layout vinculado a WebQuest deve ser um layout sem elementos cadastrados (apenas o layout básico);

3 - Caso o layout tenha ao menos um elemento, ele deve possuir todos os elementos básicos, os demais elementos relacionados a WebQuest em questão (caso haja algum além do básico) e por fim todos os elementos (caso hajam) com botões $\mathrm{A}$ e $\mathrm{B}$ cadastrados, a fim de evitar falhas no layout.

Após a publicação da Webquest, os seguintes detalhes devem ser observados:

1 - Caso um novo elemento seja inserido em uma WebQuest já publicada, e este novo elemento não possua um conteúdo cadastrado, a WebQuest será automaticamente despublicada no momento em que o usuário clicar na ação visualizar, não ficando disponível para visualização até que esta atenda as necessidades básicas da publicação, citadas acima.

2 - Caso o usuário insira o elemento e não cadastre seu conteúdo, quanto um aluno for visualizar a WebQuest ele não terá acesso ao elemento sem conteúdo.

3 - Caso seja inserido um elementos de layout no layout da WebQuest e a WebQuest não estiver vinculada ao elemento em questão, a WebQuest não será afetada, por exemplo, Se a WebQuest $\mathrm{X}$ possuir apenas os elementos básicos vinculados a ela $\mathrm{e}$ estiver cadastrada com o layout $\mathrm{Y}$ e este layout possuir os elementos básicos perfeitamente cadastrados (com botões A e B) o layout será considerado válido para a WebQuest X. Se no layout Y for inserido um novo elemento de nome "Fontes" e este elemento não for inserido na Webquest $\mathrm{X}$, a inclusão deste elemento não afetará a WebQuest X. 
Durante a validação para publicar Webquests, o WQC exibe mensagens referentes à situação da WebQuest no momento em que o usuário clica na ação publicar.

Quanto a WebQuest atender a todas as exigências do sistema, ela poderá ser publicada sem problemas. Uma vez publicada esta ficará visível para os usuários finais.

A ação "Visualizar" possibilita a visualização do resultado final da Webquest, estando ela publicada ou não. Nesta ação a WebQuest é montada dinamicamente, tendo a sua esquerda os elementos alinhados de acordo com a ordem pré-definida, tendo no topo a imagem do layout ou o nome da WebQuest, conforme o caso e por fim tendo a direita da página o conteúdo dos elementos da WebQuest. A Figura 9 ilustra uma WebQuest sendo visualizada e com o elemento "Fontes" selecionado e os elementos reordenados.

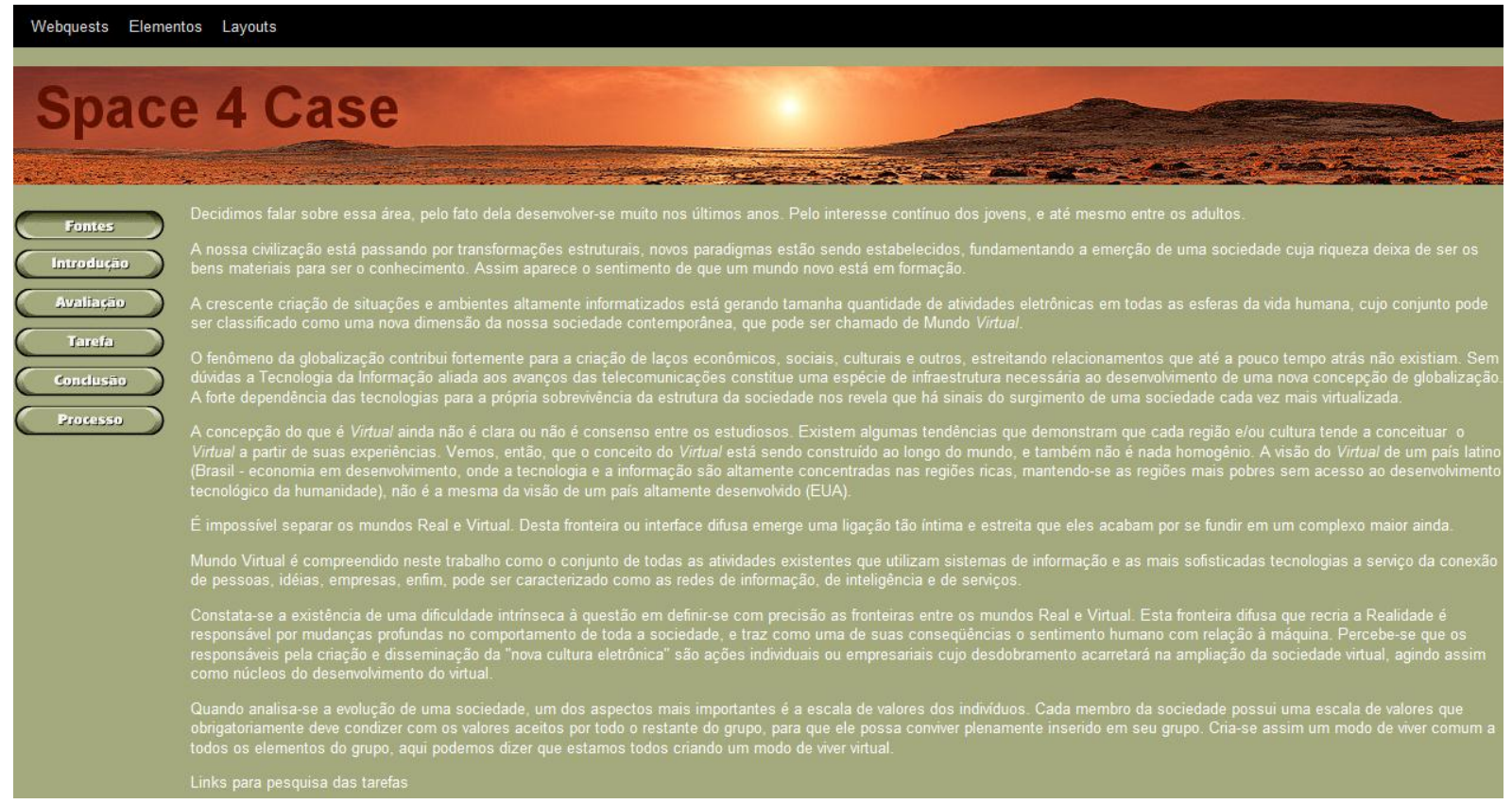

Figura 9. Visualização de webquest

Caso um usuário com privilégios acesse esta ação e a Webquest não esteja publicada, ela será exibida na forma em que se encontra, com todos os possíveis erros de layout que ainda precisam ser ajustados. Caso uma WebQuest seja alterada, esteja como publicada e com a alteração passe a não atender os critérios de uma WebQuest publicada, no momento da ação visualizar a ferramenta despublicará a WebQuest automaticamente.

A ferramenta também gerencia os usuários de acordo com o perfil dos usuários do Moodle, tendo privilégios para editar uma WebQuest apenas os usuários com privilégios do Moodle, como o Administrador e o Professor Editor. A ferramenta apenas permite edição de qualquer registro pelo próprio autor ou pelo Administrador do site.

Um usuário com o perfil de aluno terá acesso apenas a uma tabela com as Webquests publicadas para o curso que ele acessar e a posterior visualização destas.

\subsection{Implementação da Ferramenta}

A arquitetura da ferramenta segue o padrão de projeto MVC (Model-ViewController) (Freeman, 2007), sendo dividida em três camadas: Model (camada de 
modelos, referente ao banco de dados); View (referente a interface do sistema, contendo apenas layouts e templates); e Controller (responsável pela lógica e regras de negócio).

Considerando que a ferramenta é um plugin para o Moodle, a mesma foi desenvolvida visando sua total compatibilidade com este ambiente. Neste sentido, foi necessário que o desenvolvimento se desse dentro das exigências do Moodle, tanto em termos de arquitetura de arquivos, pastas e etc., até padrões de nomenclatura, arquivos obrigatórios, forma de criação e manipulação do bando de dados entre outros.

Para que a ferramenta seja instalada é necessário um servidor ou um computador doméstico, que possua um servidor Apache na versão 2.0 ou maior e um servidor PHP na versão 5.3.0 ou maior. Para o servidor, serão necessárias as bibliotecas AdoDB e Smarty para auxiliar a conexão com o banco de dados e a separar a estrutura do WQC, respectivamente. Para o usuário final basta um navegador como o Internet Explorer, o Mozilla Firefox ou o Google Chrome, e acesso a Internet para o caso de um acesso remoto ao Moodle e ao WQC, ou uma rede local, para o caso de acesso local.

\section{Considerações Finais}

Este artigo apresenta uma ferramenta para criação de Webquests, que tem por objetivo estabelecer uma padronização para a forma com que estas são criadas hoje em dia e também para facilitar e agilizar o seu desenvolvimento e customização, tornando possível a expansão e a popularização desta modalidade de atividade.

Acreditamos que os professores se sentirão estimulados a criarem suas WebQuests utilizando a ferramenta, pois terão a certeza de que elas estarão menos suscetíveis a erros de formatação e poderão ser personalizadas de acordo com a necessidade dos professores.

A fim de verificar esta hipótese, estamos iniciando a validação da ferramenta com professores que utilizam o Moodle e possuem algum conhecimento de WebQuests. Como resultado desta validação, pretendemos identificar possíveis melhorias e novas funcionalidades que atendam as expectativas dos educadores que utilizam Webquests como uma forma de ensino para seus educandos. Após a validação, o WebQuest Creator (WQC) será disponibilizado na comunidade Moodle, a fim de que professores que tenham a intenção de utilizar recursos para criação de Webquests possam usufruir das possibilidades de criação, gerenciamento e customização oferecidas por esta ferramenta.

\section{Referências}

Abar, C. A. A. P.; Barbosa, L. M. (2008) WebQuest: um desafio para o professor!. São Paulo: Avercamp.

Freeman, E. (2007) Use a cabeça! - Padrões de Projetos. Rio de Janeiro: Editora Alta Books, 2007. 397 p.

Mercado, L. P. L. ; Viana, M. A. P. (2004) Projetos Utilizando Internet: A Metodologia Webquest na Prática. Maceió. 405 p.

Miguel, V. (2010) Desarrollo de un Módulo WebQuest para la Plataforma Moodle Basado en la Especificación IMS Learning Design. In Quinto Congresso Latinoamericano de Objetos de Aprendizagem, 2010, São Paulo.

Moodle. (2011) What is Moodle? Disponível em: http $/ /$ moodle.org. Acessado: agosto, 2011 . 
Moodle Module Webquest. (2010) Disponível em: http://moodle.org/mod/data /view.php?d=13\&rid=679. Acessado: março, 2010. 